\section{LA EFICACIA COLECTIVA COMO ESTRATEGIA DE CONTROL SOCIAL DEL ESPACIO BARRIAL: EVIDENCIAS DESDE CUERNAVACA, MÉXICO}

Alfonso Valenzuela Aguilera ${ }^{1}$

\section{Resumen}

La capacidad diferencial de los vecindarios y colonias para impulsar valores compartidos entre los habitantes, así como para mantener controles sociales efectivos es un factor crucial en la variación de los niveles delictivos en las comunidades, lo cual sugiere que el fortalecimiento del tejido social así como de las capacidades comunitarias de organización resultarán en una reducción significativa de los índices delictivos actuales. En el presente trabajo se presentan los resultados de la primera Encuesta de Seguridad Ciudadana para la ciudad de Cuernavaca 2010. Con una población cercana a los tres cuartos de millón de habitantes para

\section{COLLECTIVE EFFICACY AS A SOCIAL CONTROL STRATEGY OF SPACE: EVIDENCE FROM CUERNAVACA, MEXICO}

Alfonso Valenzuela Aguilera ${ }^{1}$

\begin{abstract}
The differential capacity of neighborhoods and settlements to promote shared values among residents and exercise social control is a key factor that determines crime rates within communities. In this way, the strengthening of social fabric and organizational capacities may reduce delinquency. This paper shows the results of the first Encuesta de Seguridad Ciudadana Cuernavaca 2010. With a population of about 750,000 inhabitants, 520 surveys were conducted with a 2,8\% error rate, a response
\end{abstract}


la zona metropolitana, se aplicaron 520 encuestas para el municipio con un margen de error de 2,8\%, una distribución de respuesta del 50,0\% y un nivel de confianza del 95,0\%. El trabajo intenta demostrar la relación existente entre la percepción de inseguridad y el control social informal entre los residentes. Como parte de la metodología se construyeron cinco índices: control social del espacio, vulnerabilidad, eficacia colectiva, legalidad y sentimiento de inseguridad. Los índices están referenciados geográficamente lo que nos permite relacionar la percepción del miedo, el control social y la eficacia colectiva con el territorio urbano.

PALABRAS CLAVE: CONTROL SOCIAL, VIOLENCIA, EFICACIA COLECTIVA.

Fecha de recepción: 26.10.11

Fecha de aceptación: 23.03.12

1 México. Doctorado en Urbanismo por la Universidad Nacional Autónoma de México y Postdoctorado en Revitalización Urbana por el Instituto Francés de Urbanismo de París.

188 revista invi № 74 / Mayo 2012 / Volumen № 27: 187-215 distribution of $50 \%$ and a confidence level of 95\%. This paper demonstrates the relationship between the sense of insecurity and the informal social control among residents. Five rates were created as part of this methodology: social control of space, vulnerability, collective efficacy, legality and sense of insecurity. These rates are geographically identified, thus allowing the relationship among sense of fear, social control and collective efficacy with the urban territory.

KEYWORDS: SOCIAL CONTROL, VIOLENCE, COLLECTIVE EFFICACY

Received: 26.10.11

Accepted: 23.03.12

\footnotetext{
Mexico. Ph.D. in Urban Planning, Universidad Nacional Autóno ma de México. Postdoctorate in Urban Revitalization, Institut d'urbanisme de Paris.
} 


\section{Introducción: inseguridad y el control del territorio en México}

El control que ejerce el Estado de manera institucional se basa en acciones coercitivas que inducen a la conformidad con el orden social existente ${ }^{2}$. Dicha intervención puede extenderse hasta la cooptación, neutralización o supresión de expresiones contestatarias mediante la represión el encierro o el aniquilamiento. En la última década el control coercitivo ha sido presentado como la única alternativa para enfrentar la inseguridad creciente de las ciudades en América Latina, desestimando el control social informal, planteado como "la capacidad de la sociedad de regularse a sí misma, de acuerdo con los principios y valores acordados"3. Siguiendo a Janowitz ${ }^{4}$, el control coercitivo -que descansa predominantemente en el uso de la fuerza- resulta necesario aun en los sistemas de control social relativamente efectivos, pero limitados y circunscritos a un sistema legitimado de normas.

El Estado como garante de la seguridad pública y de un orden social establecido ha sido superado con el aumento vertiginoso del sentimiento de vulnerabilidad ciudadana ante la percepción de una amenaza potencial que no distingue lugares ni individuos. La mencionada legitimidad del orden social es la que permite sustentar los controles sociales informales como son la auto-regulación, la supervisión mutua así como las conductas rutinarias que en su momento configuran las formas sociales de convivencia. Por tanto, es importante enfatizar la diferenciación entre seguridad y control. Si bien en América Latina se presenta una simbiosis entre los dos conceptos, el control del crimen involucra tanto las acciones para el restablecimiento del orden por parte de los ciudadanos tanto en el mantenimiento de la cohesión social como en el manejo de las relaciones de grupo. No obstante, es frecuente que la atención se centre en las instituciones del Estado mientras se subestiman las prácticas sociales informales sobre las que depende la acción estatal. Las teorías que están modelando las políticas de seguridad pública se basan en los mecanismos de control que identifican al crimen y la delincuencia no como problemas de privaciones sino como resultado de la falta de mecanismos de control adecuados.

De acuerdo con Wacquant, la adopción de políticas basadas en las teorías de las "Ventanas Rotas" o los programas como "Tolerancia Cero" imponen la adopción de una aproximación coercitiva al

Cohen, 1985:15.

3 Janowitz, 1988: 84

4 Janowitz, 1991:74. 
crimen y responden a su vez a las transformaciones estructurales del Estado en marcha de los últimos años ${ }^{5}$. Mientras que las causas raíz han sido enmarcadas como un asunto de responsabilidad total del gobierno, otros sectores identifican al crimen como responsabilidad del individuo, sin ninguna consideración hacia las cuestiones estructurales como son la asimetría del poder y la riqueza, el desempleo, o bien la desaparición progresiva de los servicios sociales más elementales. Dicho autor va aún más lejos y sugiere que como resultado directo de la delegación de funciones públicas a empresas privadas, la industria del castigo ha venido a establecerse, trastrocando el sentido original del establecimiento de penas y condenas para infractores del orden social ${ }^{6}$. El impulso al mercado privado de la seguridad se relaciona con el concepto de Prison-industrial complex, en donde una red de grupos de interés relacionados con la construcción, mantenimiento y servicios ligados al sistema carcelario promueven la expansión de un Estado coercitivo y policial ${ }^{7}$.

El caso de la Ciudad de México es emblemático del fenómeno descrito toda vez que en el año de 2002 el entonces jefe de gobierno contrató al Grupo Giuliani como consultores en materia de seguridad, lo cual generó fuertes cuestionamientos sobre la

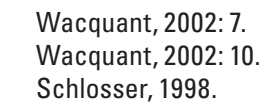

190 revista invi № 74 / Mayo 2012 / Volumen № 27: 187-215 manera de encarar un problema tan delicado como la lucha contra el crimen. Por una parte, la distancia ideológica entre los líderes no pareció afectar el modo en que el problema de inseguridad iba a ser encuadrado y por la otra, es cuestionable que los 4.3 Millones de dólares que costaría la consultoría fueran cubiertos en buena parte por Carlos Slim Helú, el empresario que encabeza la lista de Forbes y propietario de una buena parte del parque inmobiliario en el centro histórico de la ciudad, el cual existía ya como un laboratorio del modelo Neoyorquino ${ }^{8}$. Si bien las recomendaciones de Giuliani no se aplicaron a la letra como políticas públicas de manera directa, lo cierto es que tuvieron un impacto directo en la legislación vinculada con la seguridad, y sirvieron para encuadrar el problema bajo una perspectiva de atención más que a las causas, a los efectos 9 .

No obstante, el entonces Secretario de Seguridad Pública mostró cierta reserva hacia el reporte Giuliani y solicitó la asesoría de una organización no gubernamental de Abogados especializados en Derechos Humanos con sede en Nueva York ${ }^{10}$, para que emitieran una opinión especializada sobre la consultoría. Si bien la organización saludó la iniciativa de modernizar las operaciones propuestas, también advirtió que las recomendaciones no

\footnotetext{
Davis, 2007: 640.

9 Valenzuela, 2005.

10 Lawyers Committee for Human Rights, 2003.
} 
constituían un plan estratégico en sí mismas. Por una parte, destacaban el hecho de que dicho reporte presentaba una visión limitada de la participación comunitaria, sin aportar los elementos de información pública indispensable, que son claves para asegurar la confianza de la gente; mientras que por la otra, la evaluación destacaba que la propuesta del Grupo Giuliani no debería ser considerada como única opción, sino que debería quedar sujeta a un debate público significativo para hacer una estimación cuidadosa de los posibles efectos que tendría en México su aplicación. Finalmente, las recomendaciones de Giuliani fueron desestimadas como inviables para su aplicación de manera tácita, pero lo cierto es que comenzaron las ocupaciones territoriales en zonas conflictivas, se hostigaron a vendedores informales y se publicó la Ley de Cultura Cívica, la cual dejó entrever rasgos de autoritarismo detrás de dichas acciones ${ }^{11}$.

En México podemos sugerir que las tendencias globales de coerción y vigilancia se están traduciendo en el surgimiento de un Estado punitivo y policial, especialmente a partir del momento en que el presidente Calderón declaró de manera unilateral una "Guerra contra las Drogas", disponiendo para ello un presupuesto cercano a 46 mil millones de dólares en los primeros tres años de

11 Dicha Ley criminalizaba el trabajo informal y las actividades de supervivencia de la población más vulnerable y desprotegida. su administración ${ }^{12}$. Mediante una estrategia inspirada en el Plan Colombia, Calderón emprendió desde el inicio de su gobierno una guerra frontal contra algunos de los Cárteles del narcotráfico teniendo como escenario las ciudades con plazas estratégicas dentro del mercado de la droga (Tijuana, Juárez, Culiacán, Monterrey, Cuernavaca, etc.).

Uno de los aspectos que ha sido poco analizado de dicha guerra son los efectos que a nivel territorial ha significado la utilización de las ciudades como campos de batalla. Es frecuente por ejemplo, que en distintas ciudades del país se desarrollen enfrentamientos entre cárteles rivales, se hagan bloqueos de las vías principales y se realicen ocupaciones militares en conjuntos residenciales de lujo o en fraccionamientos exclusivos cerrados, transgrediendo el espacio urbano. Ante este panorama, algunos sectores de la población en Cuernavaca vieron con beneplácito la substitución de los elementos de la policía local y estatal por personal del ejército ya desde hace más de una década, si bien en la actualidad esta medida se ha revelado como un agravante dentro de las condiciones imperantes. Por una parte, la militarización de las fuerzas policíacas estuvo basada en el supuesto que sólo el ejército podría recuperar el orden y la disciplina en la corporación. Sin embargo, un régimen militarizado generalmente responde a los sectores

12 Arteaga y Jiménez, 2010. 
autoritarios de la sociedad, para quienes el control social es aplicado mediante medios coercitivos en lugar de por procesos de negociación. Por otra parte, mientras que la policía reconoce en el infractor a un ciudadano que está actuando fuera de la Ley y es necesario sancionarlo para enmendar la falta, la visión militar identifica a un enemigo -que no ciudadano- al que hay que neutralizar o eliminar. No es entonces extraño que las estrategias utilizadas en el combate al crimen de los últimos años se basen en operativos "relámpago" (blitzing), razzias y "ocupaciones" del territorio, en donde dichos operativos muchas veces son detonados por eventos que han provocado la indignación popular, en donde la racionalidad subyacente es que dadas las extensivas redes de corrupción y complicidades en todos los niveles de la estructura de mando es necesario actuar de manera total e impredecible.

Existe toda una discusión acerca de la legitimidad de la violencia, desde Wright Mills definiendo el Estado como "el dominio de un hombre sobre otro basado en la violencia legítima, o supuestamente legítima"13 , hasta Max Weber y León Trotsky quienes compartían la visión de un Estado que está siempre fundado sobre la violencia. Al respecto Arendt matiza el hecho de que si bien el poder efectivamente es la esencia de todo gobierno, la

13 Wright Mills, 1956:171.

192 revista invi № 74 / Mayo 2012 / Volumen № 27: 187-215 violencia no lo es. Por tanto, al tener la violencia una naturaleza instrumental el Estado la utiliza como medio para la consecución de sus fines ${ }^{14}$. En el caso del México contemporáneo, la violencia ha sido instrumental para tratar de ganar legitimidad, después de una elección presidencial fuertemente cuestionada en términos de transparencia y legalidad. Más adelante, la violencia desatada a partir de la declaración de guerra contra el narcotráfico generó un replegamiento de los ciudadanos a la esfera privada mientras el ejército y las fuerzas del orden pasaron a cumplir funciones policiales dentro de la esfera pública. Si validamos la propuesta de que la violencia es instrumental, siempre necesitará justificarse a través de los fines que persigue, y si bien el ejercicio del poder no necesita justificación, sí requiere un cierto grado de legitimidad ${ }^{15}$.

\section{La percepción del miedo y su impacto en la ciudadanía}

Desde el punto de vista de la sicología, el miedo al crimen puede ser visto como un estado o como una disposición ${ }^{16}$. Mientras el miedo al crimen como estado es una emoción multidimensional, la disposición refiere una tendencia a experimentar el miedo en repetidas ocasiones ante la idea de

14 Arendt, 1969:51.

15 Arendt 1969:52.

16 Ute y Greve, 2003 
convertirse en víctima de un crimen. El presente trabajo busca iluminar la distribución social y espacial del miedo en la ciudad para poder monitorear su impacto sobre las comunidades. El miedo parece estar intensificando la sensación de vulnerabilidad, la cual va en detrimento de la disposición de los individuos para interactuar socialmente. De acuerdo con Wurff y Stringer ${ }^{17}$, el estado del miedo es "la percepción de una amenaza hacia algún aspecto del bienestar que coincide con la sensación de ser incapaz de enfrentar el reto". Si el control social es un atributo de cohesión comunitaria, la cual permite ejercer un cierto control sobre la propia vida así como sobre el comportamiento de otros, la gente puede intensificar su miedo al constatar que no cuenta con el soporte social necesario para apelar a las normas de comportamiento sobreentendidas por una comunidad determinada.

Existe una distinción que queremos introducir con respecto al concepto de inseguridad en donde -ya sea como percepción o como sentimiento- el producto de esta inseguridad es en realidad lo que causa el mayor de los conflictos al individuo: la vulnerabilidad. Definida como la incapacidad para hacer frente a un riesgo inminente, la vulnerabilidad es definida por Chambers como "la exposición a contingencias y tensión, y la dificultad para

17 Wurff, A. van Der, Stringer, P., and Timer F. (1988). afrontarlas. La vulnerabilidad tiene por tanto dos partes: una parte externa, de los riesgos, convulsiones y presión a la cual está sujeto un individuo o familia; y una parte interna, que es la indefensión, esto es, una falta de medios para afrontar la situación sin pérdidas perjudiciales" ${ }^{18}$.

Si bien la vulnerabilidad es una dimensión relativa -dado que todas las personas somos vulnerables-, estamos expuestos a tres tipos de riesgos: el riesgo de exposición a las crisis o convulsiones; el riesgo de una falta de capacidad para afrontarlas; y el riesgo de sufrir consecuencias graves a causa de ellas, así como de una recuperación lenta o limi$\operatorname{tada}^{19}$. El sentimiento de vulnerabilidad /inseguridad se alimenta además de las imágenes mediáticas que devuelven a la sociedad un imaginario violento de la lucha contra un enemigo interno (el narcotráfico, en el caso de México), el cual es imperioso controlar pero que, sin embargo, se revela más poderoso que el Estado, contribuyendo así a su descrédito. Sin embargo, más que un objeto al cual temerle, es la capacidad de enfrentarlo o controlarlo lo que genera angustia y desasosiego. De acuerdo con Kaufman, mientras que el miedo olvida a un objeto determinado, la angustia constituye "una reacción ante el peligro cuando éste no puede ser identificado o definido con claridad"20.

18 Chambers, R. (1989:5)

19 Bohle, H. G.; T. E. Downing y M. J. Watts, 1994: 37-48.

20 Kaufman S., 2006. 
En áreas con índices delictivos elevados existe sin embargo, una marcada tendencia de sus habitantes por mantener de manera informal un control sobre las actividades delictivas en su territorio. Por ejemplo, en barrios dominados por pandillas, algunos de los líderes participan en la reducción de signos de desorden (graffiti, vandalismo, prostitución, robos) que atraigan atención policial negativa hacia su vecindario ${ }^{21}$. En la presente investigación encontramos que el control social informal es un proceso dinámico, el cual es activado de manera diferencial en las distintas colonias en donde fue analizado. De acuerdo con Felson, en los sistemas urbanos modernos los residentes atraviesan los límites territoriales de distintos barrios o colonias a lo largo del día, sin embargo, nos interesan estas últimas como unidades en donde se experimenta un control sin importar de donde provengan los delincuentes ${ }^{22}$.

La evidencia presentada en el presente estudio muestra que es posible medir el nivel de eficacia colectiva y control social del espacio a nivel de colonias y establecer su relación mediante correlaciones simples. la eficacia colectiva -definida como la cohesión social aunada a una expectativa común de control social del espacio- incide significativamente en la percepción de la seguridad en el territorio. Del mismo modo, el control social

21 Patillo Mary E. 1988.

22 Felson, Marcus, 1987: 911-31. del espacio representa un elemento central en la percepción del peligro y, aun en zonas con altos niveles delictivos, dicho control ayudó a mantener bajos niveles de inseguridad en el territorio.

De acuerdo con Earls ${ }^{23}$, las decisiones que toman los residentes de una comunidad para defender los recursos de la colectividad en caso necesario, inciden de manera significativa tanto en la disminución de los índices criminales como en la percepción misma de la inseguridad. Por tanto, la teoría de las "ventanas rotas" no ha encontrado una correlación definitiva con el aumento del crimen. En palabras de uno de los creadores de dicha teoría "[...] aun hasta el día de hoy no estoy seguro de que mejorar el orden reduzca o no el crimen" ${ }^{24}$. Si bien los signos del desorden en la ciudad (graffiti, basura, entornos degradados) pudieran contribuir en el deterioro del tejido social, las evidencias en este estudio apuntan a que factores como la cohesión social, la legalidad y la eficacia colectiva sean determinantes para definir la percepción de inseguridad de los residentes y el control que pueden ejercer en el territorio. Lo anterior es relevante toda vez que muchos gobiernos han optado por endurecer las penas carcelarias, aumentar la represión y discrecionalidad policial o bien utilizar estrategias militares en zonas urbanas. El resultado ha sido la pérdida de confianza en el sistema de impartición 
de justicia e incluso un sentimiento de vulnerabilidad extensiva por parte de la ciudadanía, lo cual ha derivado en que las acciones de reacción y represión hayan cobrado un rol preponderante como respuesta a la seguridad en lugar de la prevención situacional del crimen.

En casos exitosos de reducción de los índices de criminalidad alrededor del mundo (como puede ser Bogotá o Medellín), se ha demostrado que es necesaria la colaboración de distintas agencias de gobierno para atacar el crimen tanto como las desventajas sociales, la exclusión y segregación, lo cual permita la creación de oportunidades y mejores condiciones de vida. Más aún, el enfoque estrecho de la prevención del crimen se está transformando en una cuestión más amplia como la seguridad comunitaria así como el convertirla tanto en un derecho humano básico como en un bien común. Al respecto existen distintas iniciativas para superar las condiciones económicas y sociales que detonan el crimen y la victimización, además de, por otra parte, el reconocimiento de que los gobiernos, las comunidades y las fuerzas del orden pueden establecer asociaciones y colaboraciones para la construcción compartida de la seguridad.

Si bien algunos gobiernos empiezan a considerar tanto al crimen como al narcotráfico como un problema de salud y calidad de vida más amplio, en ciudades en donde se registra una alta tasa de mortalidad ligada al crimen existen ahora generaciones de niños que están creciendo sin padres -y con el creciente índice de encarcelamiento femenino, sin madres-, dejándolos sin cuidados parentales. Como resultado, la red social conformada por el círculo comunitario inmediato en su rol de padres, amigos o vecinos, se ha venido desarticulando, dejando desprotegidos los controles sociales informales. El espacio es entonces crucial para entender la inseguridad: en los Estados Unidos cincuenta por ciento de los crímenes violentos ocurren en tres por ciento del territorio, lo cual nos habla de una clara geografía del crimen focalizada en ciertos puntos. En el caso mexicano tenemos que tanto los victimarios como las víctimas provienen generalmente de los mismos barrios o comparten una misma condición socioeconómica ${ }^{25}$.

Analizando los costos económicos y sociales del crimen encontramos que los métodos coercitivos contra el crimen utilizados de manera generalizada, aparte de ser costosos, no resuelven las consecuencias a largo plazo para las familias involucradas, ni tampoco ayudan a prevenir la delincuencia o la reincidencia futura. La prevención es hasta diez veces más efectiva en relación a su costo que las medidas tradicionales como la encarcelación y aporta otros beneficios tales como mejoría

25 De acuerdo con una encuesta Británica 4 por ciento de las víctimas sufren 40 por ciento de los crímenes. 
en educación, capacitación laboral y salud. En algunas ciudades como Medellín, Río de Janeiro o Curitiba se han combinado intervenciones de renovación y acondicionamiento urbano con acciones que buscan fortalecer las capacidades de los residentes para integrarse mejor a la sociedad con resultados altamente positivos ${ }^{26}$.

Las intervenciones sin embargo, tienen que ir más allá de proyectos aislados para las familias que viven en áreas con altos índices delictivos y en cambio, tanto el apoyo como la coordinación de los distintos niveles de gobierno desde el nacional regional, estatal y local es crucial para el acoplamiento de las acciones. Para ello, se requiere de un verdadero compromiso y liderazgo de personajes de alto nivel que ubiquen los asuntos relativos a la seguridad comunitaria como prioridad dentro de las agendas políticas. Si bien no podemos considerar las comunidades, vecindarios o colonias como entes homogéneos, al reconocer que dichos núcleos están compuestos de grupos de interés, muchas veces en conflicto, nos permitirá poner las cartas sobre la mesa de manera abierta y transparente. Dentro del modelo económico actual es indiscutible que han aumentado el número de pobres y que cada vez aumenta la polarización socioeconómica global ${ }^{27}$, sin embargo, la promoción de la

26 Arriagada y Godoy, 1999

27 Firebaugh, G., 2003. seguridad comunitaria, la reducción del crimen es considerada como un asunto de buena gobernanza, que requiere la vinculación de la comunidad en asociaciones $^{28}$.

\section{Las herramientas de control social y eficacia colectiva}

La espacialización de las actividades criminales nos permite identificar distintos factores por los cuales existe una concentración de violencia interpersonal en ciertas partes de la ciudad. Sin embargo, no son del todo evidentes los vínculos entre las características físicas de un vecindario y los índices de criminalidad registrados por la policía local. Para el caso de la ciudad de Cuernavaca, si bien es cierto que los vínculos sociales no se circunscriben única y exclusivamente a los límites de las colonias, lo cierto es que existe un fuerte sentido de pertenencia especialmente en las colonias clasificadas como de alto riesgo. Otro factor que puede incidir significativamente en la percepción de la inseguridad y la sensación de vulnerabilidad es la existencia de asociaciones de vecinos que pueden o no estar relacionados con el combate a la inseguridad como procesos locales que pueden

28 Gobernanza es el concepto de reciente difusión para designar a la eficacia, calidad y buena orientación de la intervención de Estado, que proporciona a éste buena parte de su legitimidad en lo que a veces se define como una nueva forma de gobernar. 
afectar la percepción de inseguridad. Sin embargo, en este trabajo optamos por incluir la pertenencia a asociaciones vecinales dentro de un indicador más completo y que incluye una esfera más amplia en donde se desenvuelve el individuo en su vida cotidiana, ligados a procesos y dinámicas locales del crimen. Por tanto, integramos dichas dimensiones del entorno urbano dentro de un encuadramiento teórico que destaca el papel del control social y la eficacia colectiva como indicadores que inciden en la percepción de la inseguridad, mitigan el sentimiento de vulnerabilidad y explican su relación con el crimen real.

Si bien la desorganización social ha sido definida como la incapacidad de una estructura comunitaria para reconocer valores comunes entre sus residentes y mantener entonces controles sociales efectivos $^{29}$, encontramos que en el caso de barrios considerados como peligrosos existen complejos sistemas de vinculación, amistades y complicidades, pero esto no implica necesariamente que se compartan los mismos valores por la generalidad. En entornos con altos niveles delictivos también se mantienen relaciones de poder bien establecidas en donde la divergencia o la falta a los códigos de silencio pueden traer consecuencias fatales o indeseables para los trasgresores.

29 Bursik, 1988; Kornhauser, 1978.
Por otra parte, se ha establecido en la literatura comunitaria el concepto de capital social, en donde a través de experiencias colectivas se crean vínculos entre personas y posiciones. De acuerdo con Putnam, el capital social contiene "las características propias a la organización social tales como redes, normas y vínculos de confianza, las cuales facilitan la coordinación y la cooperación para un beneficio mutuo" 30 . Sin embargo, en los barrios referidos el capital social podría estar también relacionado con procesos sociales al margen de la ley y que sin embargo se encubren mediante redes sociales, vínculos interpersonales y un control social informal del espacio que funciona de manera altamente efectiva para mantener la zona con un nivel de riesgo controlado ${ }^{31}$.

Por otra parte, en contextos de alta incidencia delictiva pueden existir estrechos vínculos interpersonales, redes sociales consolidadas, o bien, una buena integración social y no obstante, tener mecanismos de control social débiles o pocas posibilidades de incidir en los niveles de seguridad de la comunidad $^{32}$. Es importante destacar el punto que refiere Sampson sobre la necesaria relación de los vecindarios con las dinámicas urbanas extensivas a la ciudad: "[... s si el capital social es verdaderamente relacional, entonces las investigaciones que consideran

30 Putnam, 1993:36.

31 Patillo-Mc Coy, Mary E, 1999.

32 Wilson, 1996 
los vecindarios como islas dentro de sí mismas, están perdiendo de vista el punto teórico principal"33. Existe una propuesta desde la economía política que sugiere que los vecindarios son interdependientes entre sí, de modo que existe un impacto no solo a nivel de oportunidades, sino que la cercanía a zonas deterioradas o violentas puede llegar a ser aun más determinante que las características estructurales mismas de la comunidad ${ }^{34}$. A este respecto podemos referirnos a la definición de control social como la capacidad de una unidad social para auto-regularse de acuerdo con objetivos y principios colectivos distintos a un conformismo forzado ${ }^{35}$.

Utilizando el concepto propuesto por Sampson ${ }^{36}$, la eficacia colectiva interrelaciona la cohesión social y la confianza mutua con la disposición compartida por los residentes para intervenir en el mantenimiento de un control social en el territorio. La eficacia colectiva es instrumental para obtener un efecto determinado e implica un sentido de compromiso proactivo que la comunidad asume como sobreentendido. Si bien algunos teóricos de la economía política han sugerido que debemos considerar modelos que incorporen las dinámicas de la ciudad en sentido extenso ${ }^{37}$, también es cierto que la espacialización de las actividades criminales, así como su

33 Sampson, Morenoff y Earls, 1999: 637.

34 Morenoff y Sampson, 1997: 31-64.

35 Janowitz, M. 1975.

36 Sampson, Raudenbush y Earls. 1996:918-924.

37 Logan y Molotch, 1987. localización en el territorio nos permiten identificar las dinámicas locales que explican la vulnerabilidad espacial de los habitantes de zonas específicas ${ }^{38}$.

A este respecto, es importante la distinción que establece Bauman con respecto a la comunidad como esa especie de paraíso perdido en donde imaginamos un núcleo social onírico y seductor y la acepción de la comunidad como lo realmente existente, en donde el "estar en comunidad" demanda un precio que a veces conlleva a la pérdida de un grado de libertad considerable ${ }^{39}$. Existen entonces en juego tres elementos definitorios: la comunidad, la libertad y la seguridad. Por tanto, si para obtener la seguridad que proporciona el vivir en comunidad es necesario sacrificar la libertad, nos encontramos ante una disyuntiva que amerita establecer un equilibrio entre dichos componentes para alcanzar una vida plena. En el presente estudio encontramos correlaciones positivas entre los vínculos sociales entre los residentes ligados al territorio y un menor sentido de vulnerabilidad e inseguridad con respecto al territorio.

De acuerdo con Bandura ${ }^{40}$, la percepción que tiene la gente con respecto a su capacidad para controlar los eventos que afectan sus vidas incide sobre sus decisiones, sus aspiraciones, percepción sobre la 
propia vulnerabilidad, así como su disposición a intervenir para lograr cambios significativos. El sentimiento de inseguridad o de vulnerabilidad ha cobrado importancia en los últimos años porque, al ser de cierto modo independiente del delito real puede canalizarse como movilizaciones colectivas en contra de un gobierno que pierde legitimidad. Aun en casos donde se ha reducido los índices delictivos, es común encontrar que el sentimiento de vulnerabilidad o la percepción de inseguridad aumenten incluso entre los sectores de la población menos propensos a sufrir un delito, como las mujeres y los ancianos (el 89\% de la víctimas son hombres entre 20-30 años de edad). Sin embargo, de acuerdo con Goodey las mujeres tienden a expresar su temor y sentir abiertamente su vulnerabilidad al crimen, mientras que los hombres tienden a disimular el miedo, ocultarlo o transportarlo en ira ${ }^{41}$. En nuestra encuesta se manifestó de manera considerable que las mujeres percibían una mayor inseguridad, declararon sentirse más vulnerables y en general más sensibles al deterioro social y espacial de la comunidad.

Existen distintas perspectivas teóricas que identifican a las comunidades como densas redes de vínculos sociales a la manera de "aldeas urbanas". Si bien los vecindarios contemporáneos se caracterizan por la existencia de vínculos sociales débiles así como la extraterritorialidad de las relaciones sociales, en

41 Goodey Jo (1997). el caso de Cuernavaca existe una identidad relativamente fuerte en las colonias, en algunos casos debido a su origen popular, en otros por haber derivado de pueblos antiguos (Centro, Ocotepec) y en general por ser colonias con larga tradición comunitaria. Como parte de las cuestiones fundamentales para explicar la relación entre los componentes estructurales del entorno físico comunitario y los índices de crimen violento podrían relacionarse con la eficacia colectiva y el control social del espacio. Es importante sin embargo, identificar procesos extra-locales relacionados con las dinámicas espaciales del crimen violento. Cuernavaca es un lugar en donde los últimos dos años ha habido una escalada del crimen y la componente espacial ha sido central en el desarrollo de zonas de riesgo.

La presente investigación toma como punto de partida los planteamientos surgidos a partir de la década de los setenta, en donde se define la teoría de la "desorganización social", entendida como la inhabilidad de una estructura comunitaria para reconocer valores comunes entre los residentes, lo que les permite ejercer controles sociales efectivos $^{42}$. De acuerdo con Bursik los vecindarios carentes de capital social o redes sociales interdependientes, tienen menos posibilidades de identificar valores comunes así como mantener el control social informal del espacio ${ }^{43}$.

42 Bursik, 1988, Kornhauser, 1978; Sampson and Groves, 1989.

43 Bursik, 1988. 


\section{Una aproximación metodológica: la construcción de indicadores}

La Primera Encuesta de Cultura Ciudadana de Cuernavaca $2010^{44}$ se diseñó con un muestreo de tipo bietápico estratificado por conglomerados y se calculó con un margen de error del 2,75\% y un nivel de confianza del 95,0\%, estando representadas las ocho delegaciones que componen el Municipio de Cuernavaca. La encuesta aborda siete temas relevantes para la problemática: 1) Eficacia Colectiva; 2) Control Social informal del espacio urbano; 3) Percepción de Inseguridad; 4) Estado de Derecho; 5) Género e Inseguridad; 6) Victimización; 7) Cohesión Familiar. A partir de dichas secciones se construyeron los indicadores de control social informal y de eficacia colectiva para determinar su respectiva correlación con la percepción de inseguridad en el territorio. En una segunda fase, dichos indicadores se relacionarán tanto con los índices delictivos reportados por la Municipalidad en cada colonia para después ser georreferenciados a nivel colonia.

La encuesta se enfocó a la percepción que la población tiene sobre la seguridad urbana en Cuernavaca, a principios de 2010. La muestra estuvo integrada

44 La Primera Encuesta de Cultura Ciudadana de Cuernavaca 2010 forma parte fundamental del proyecto CONACYT de Ciencia Básica \# 78820 titulado "Espacio público, Eficacia Colectiva y Control Social del Espacio" (2009-2012) bajo la dirección del autor. por 500 encuestas levantadas en viviendas de 14 colonias claves, que tanto por su ubicación como por la percepción empírica de la inseguridad en dichas demarcaciones. En la tabla 1.0 se presentan datos sobre la muestra seleccionada, basada en la población base y número de viviendas, producto del II Conteo de Población y Vivienda 2005 (INEGI).

\section{TABLA 1 MUESTRA SELECCIONADA PARA LA PRIMERA ECC2010}

\begin{tabular}{llrr} 
No. Colonia & $\begin{array}{r}\text { Total de } \\
\text { Viviendas 2005 }\end{array}$ \\
\hline 1 & Acapantzingo & 930 & 30 \\
\hline 2 & Altavista & 1466 & 48 \\
\hline 3 & Antonio Barona Centro & 2387 & 78 \\
\hline 4 & Antonio Barona secc. 1 & 1017 & 33 \\
\hline 5 & Antonio Barona secc. 2 & 144 & 5 \\
\hline 6 & Antonio Barona secc. 3 & 628 & 20 \\
\hline 7 & Centro & 3113 & 102 \\
\hline 8 & Chapultepec & 1357 & 44 \\
\hline 9 & Chulavista & 483 & 16 \\
\hline 10 & Flores Magón & 291 & 10 \\
\hline 11 & Flores Magón 1er Fracc. & 572 & 19 \\
\hline 12 & Flores Magón 2do Fracc. & 970 & 32 \\
\hline 13 & Flores Magón 3er Fracc. & 337 & 11 \\
\hline 14 & Ocotepec & 1610 & 52 \\
\hline Total de Viviendas & 15305 & 500
\end{tabular}

Fuente: Elaboración propia con base en el II Conteo de Población y Vivienda (INEGI, 2005). 


\section{METODOLOGÍA PARA LA CONSTRUCCIÓN DE ÍNDICES Y GRADOS}

Se construyeron tres índices, con base en las respuestas proporcionadas por la población encuestada:

1. Índice de percepción de inseguridad

2. Índice de eficacia colectiva

3. Índice de control social del espacio

Con la suma y peso relativo de cada una de las preguntas, se calculó un índice compuesto cuyos valores oscilan entre el 0,00 y 1,00. Una vez calculado se le asignó un grado de estratificación con los siguientes valores:

- MUY ALTO: cuando el índice oscilaba entre 0,76 y 1,00

- ALTO: cuando el valor del índice estaba en el rango de 0,51 y 0,75

- MEDIO: cuando el valor que arroja el índice se encuentra entre 0,26 y 0,50

- BAJO: cuando el valor del índice se encuentra entre 0,00 y 0,25

1. Indice de percepción de inseguridad. El índice de percepción de inseguridad se construyó como índice al integrar las preguntas relativas a su experiencia como usuario del espacio urbano (si frecuenta zonas peligrosas, si ha cambiado de hábitos) o la manera como identificar los distintos lugares de la ciudad (cuáles sitios considera peligrosos, cuáles las colonias mas inseguras) o bien, su opinión sobre el control social formal (la policía, si está de acuerdo con la presencia del ejército o su opinión sobre el sistema de justicia). Si bien la percepción de inseguridad se relaciona con la eficacia colectiva, en particular con el sentido de comunidad que existe en los vecindarios, otros autores sostienen que el apego emocional, es decir, el componente afectivo de la cohesión social, es uno de los elementos más importantes para el control del territorio. De este modo, tanto los vínculos sociales entre la comunidad como entre las distintas generaciones fortalecen la confianza mutua y con ello mejoran la percepción general sobre la seguridad en el vecindario ${ }^{45}$.

2. Índice de eficacia colectiva. Partiendo de la definición acuñada por Sampson, la eficacia colectiva combina la cohesión social comunitaria con la disposición de intervenir en función del bien común ${ }^{46}$. Por otra parte, el sentido comunitario o la cohesión social se consideran elementos sustanciales para mitigar el sentimiento de inseguridad o el miedo al crimen ${ }^{47}$. Entre los indicadores utilizados para construir el indice de eficacia colectiva destacan la

45 Greenberg, Rohe y Williams, 1982.

46 Sampson, Raudenbush y Earls, 1997:918.

47 Greenberg, Rohe y Williams, 1982. 
identificación de vínculos comunitarios (confianza en los vecinos, ayuda mutua, sociabilidad, altruismo) y por otra parte la disposición a intervenir en situaciones de riesgo (mantenimiento de normas sociales, ayuda al prójimo, etc.), y finalmente al apego a las normas de legalidad y convivencia.

3. Indice de control social informal. El control social del espacio está planteado como la efectividad de los mecanismos informales de control que los residentes utilizan para mantener el orden público. La intervención de los residentes para impedir actos vandálicos o incivilidades es un indicador de control social informal, del mismo modo que la capacidad de controlar procesos grupales y signos visibles de desorden se consideran cruciales para reducir las oportunidades de cometer delitos en los vecindarios. En el presente estudio se utilizaron como variables para la construcción del índice de control social informal aspectos relacionados con la interacción social (confianza en los vecinos, uso de espacios públicos locales para socializar, reunirse para resolver problemas comunes), apropiación del espacio urbano (actuar al detectar grupos de riesgo, defender los espacios públicos, pertenencia a la colonia) y su disposición a participar en el cuidado y mejora de su vecindario.

202 revista invi № 74 / Mayo 2012 / Volumen № 27: 187-215

\section{FRECUENCIA DE LOS GRADOS DE CONTROL SOCIAL, EFICACIA COLECTIVA E INSEGURIDAD}

El primer paso para encontrar la distribución y el comportamiento de los tres índices calculados, posterior a su agrupación en cuatro categorías: muy alto, alto, medio y bajo, es observar su comportamiento simple en las tablas de frecuencia, tal como se muestra en la tabla $1^{48}$.

Para los tres grados calculados, se comprueba que se incluyen los 15.305 casos considerados para la población base. Ahora bien, si se observa con detenimiento se concluye que el $74 \%$ de la población encuestada percibe que se tiene en la ciudad de Cuernavaca un control social "medio", mientras que un 20\% de la población encuestada opinó que el control social en Cuernavaca es "alto", y apenas un $6 \%$ percibe un control social "bajo". En cuanto a la eficacia colectiva en la ciudad de Cuernavaca, se observa que un 56\% de la población encuestada percibe un grado "medio", seguido de un 41\% de la población que percibe una "alta" eficacia colectiva y apenas un $2 \%$ percibe una "baja" eficacia colectiva, mientras que poco más de $1 \%$ opinó que en Cuernavaca existe una "muy alta" eficacia colectiva. En lo que respecta al eje de la seguridad, captado mediante su opuesto concepto que es la

48 Cabe destacar que en el caso del grado de Control Social el programa estadístico no incluyó la categoría de Muy Alto debido a que no encontró frecuencias por lo que no se incluye en el cuadro de salida. 


\section{TABLA 2 FRECUENCIA DE LOS GRADOS DE CONTROL SOCIAL, EFICACIA COLECTIVA E INSEGURIDAD}

\begin{tabular}{|c|c|c|c|}
\hline Grado de Control Social & Frecuencia & Porcentaje & Porcentaje acumulado \\
\hline Alto & 3097 & 20.2 & 20.2 \\
\hline Medio & 11291 & 73.8 & 94.0 \\
\hline Bajo & 917 & 6.0 & 100.0 \\
\hline Total & 15305 & 100.0 & \\
\hline Grado de Eficiencia Colectiva & Frecuencia & Porcentaje & Porcentaje acumulado \\
\hline Muy alto & 185 & 1.2 & 1.2 \\
\hline Alto & 6249 & 40.8 & 42.0 \\
\hline Medio & 8592 & 56.1 & 98.2 \\
\hline Bajo & 279 & 1.8 & 100.0 \\
\hline Total & 15305 & 100.0 & \\
\hline Grado de Inseguridad & Frecuencia & Porcentaje & Porcentaje acumulado \\
\hline Muy alto & 62 & 0.4 & 0.4 \\
\hline Alto & 7307 & 47.7 & 48.1 \\
\hline Medio & 7230 & 47.2 & 95.4 \\
\hline Bajo & 706 & 4.6 & 100.0 \\
\hline Total & 15305 & 100.0 & - \\
\hline
\end{tabular}

Fuente: Elaboración propia. SPSS Ver. 19. con base en la Primera Encuesta sobre Cultura Ciudadana 2010, Cuernavaca, Mor.

inseguridad, se obtuvieron porcentajes casi similares, con los que se observa que en la ciudad de Cuernavaca, $47 \%$ de la población encuestada percibe una alta inseguridad; casi este mismo porcentaje otorgó un grado de inseguridad "medio" para la zona de estudio; mientras que casi un $5 \%$ opinaron que la inseguridad es baja.
Los comportamientos antes descritos se pueden observar mejor en la gráfica 1.0, con la que se puede concluir que la percepción de la población encuestada en cuanto a inseguridad, eficacia colectiva y control social del espacio se concentra básicamente en alto y medio. Por lo anterior, es necesario contrastar el comportamiento de crecimiento 


\section{FIGURA 1, GRADOS DE CONTROL SOCIAL, EFICACIA COLECTIVA E INSEGURIDAD}

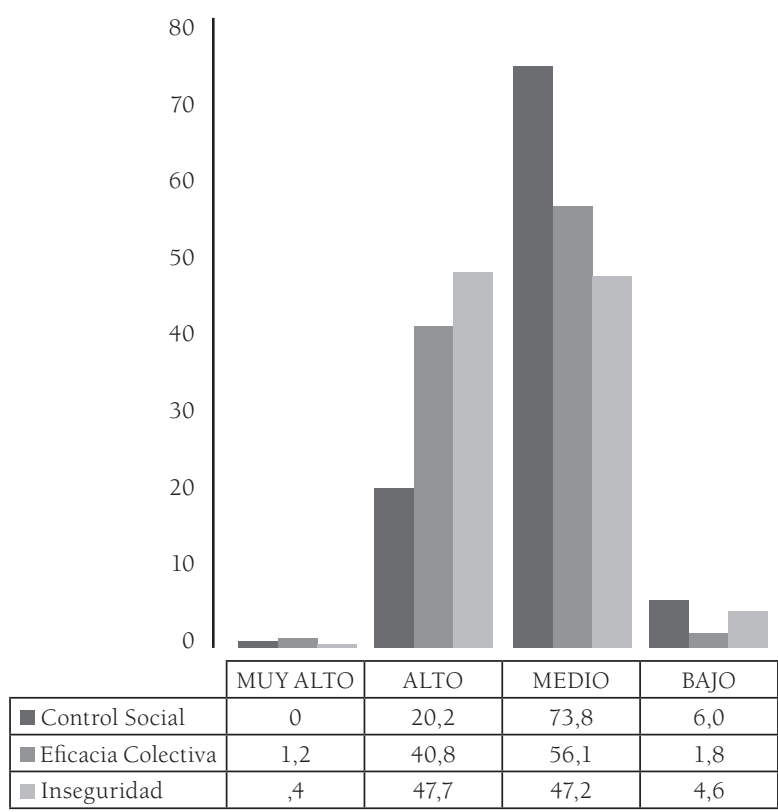

Fuente: Elaboración propia. SPSS Ver. 19. con base en la Primera Encuesta sobre Cultura Ciudadana 2010, Cuernavaca, Mor

y decrecimiento entre estos tres índices construidos y ver las interrelaciones entre ellos. Por la naturaleza teórica y empírica que estos tres índices representan para la presente investigación, se contrasta la percepción de inseguridad con el control social del espacio y posteriormente la percepción de inseguridad con la eficacia colectiva. Antes de comprobar el nivel de asociación, mediante las correlaciones, se aplica un test de chi-cuadrada ( $r$ de Pearson) para encontrar la representatividad en relación a la robustez de la encuesta acorde a las frecuencias observadas, es decir, si las diferencias presentadas son estadísticamente significativas, partiendo del supuesto (hipótesis nula) de que no hay relación entre la inseguridad y el control social, así como entre la inseguridad y la eficacia colectiva; esta medida se hará en base al grado calculado, ya que la metodología establece que esta prueba se realiza con valores nominales que representan grupos o categorías de una variable.

En la tabla 3, se puede observar una tabla de contingencia entre el grado de inseguridad y el grado de control social del espacio, en el cruce de los renglones y columnas se ilustran los valores observados de las frecuencias y los esperados. Cabe mencionar que los valores esperados son calculados mediante el procesamiento de los datos en el programa Statistical Package for the Social Sciences (SPSS), en base al tamaño de la muestra, a las frecuencias, a valores teóricos y bases que el modelo de r de Pearson implica. En el comportamiento de la inseguridad, se puede leer que de las viviendas encuestadas sólo 62 opinaron que se vive una inseguridad muy alta de las cuales 31 opinaron que se tiene un grado medio de control social del espacio y 31 de igual manera percibieron un bajo control social del espacio. 
TABLA 3 GRADO DE INSEGURIDAD ^ GRADO DE CONTROL SOCIAL

\begin{tabular}{|c|c|c|c|c|c|}
\hline \multicolumn{2}{|c|}{ GRADO DE INSEGURIDAD } & \multicolumn{3}{|c|}{ GRADO DE CONTROL SOCIAL INFORMAL } & \multirow[t]{2}{*}{ TOTAL } \\
\hline & & ALTO & MEDIO & BAJO & \\
\hline \multirow{2}{*}{ MUY ALTO } & Recuento & 0 & 31 & 31 & 62 \\
\hline & Frecuencia esperada & 12.5 & 45.7 & 3.7 & 62.0 \\
\hline \multirow{2}{*}{ ALTO } & Recuento & 185 & 6298 & 824 & 7307 \\
\hline & Frecuencia esperada & 1478.6 & 5390.6 & 437.8 & 7307.0 \\
\hline \multirow{2}{*}{ MEDIO } & Recuento & 2267 & 4901 & 62 & 7230 \\
\hline & Frecuencia esperada & 1463.0 & 5333.8 & 433.2 & 7230.0 \\
\hline \multirow{2}{*}{ BAJO } & Recuento & 645 & 61 & 0 & 706 \\
\hline & $\begin{array}{l}\text { Frecuencia esperada } \\
\end{array}$ & 142.9 & 520.8 & 42.3 & 706.0 \\
\hline \multirow{2}{*}{ TOTAL } & Recuento & 3097 & 11291 & 917 & 15305 \\
\hline & Frecuencia esperada & 3097.0 & 11291.0 & 917.0 & 15305.0 \\
\hline
\end{tabular}

Fuente: Elaboración propia. SPSS Ver. 19. con base en la Primera Encuesta sobre Cultura Ciudadana 2010, Cuernavaca, Morelos.

TABLA 4 PRUEBAS DE CHI CUADRADO CONTROL SOCIAL E INSEGURIDAD

\begin{tabular}{lrrrr}
\hline PRUEBAS DE CHI CUADRADO & \multicolumn{1}{c}{ VALOR } & GL & \multicolumn{2}{c}{ SIG. ASINTÓTICA BILATERAL } \\
Chi-cuadrado de Pearson & $4851,127^{\mathrm{a}}$ & 6 & .000 \\
\hline Razón de verosimilitudes & 4938.243 & 6 & .000 \\
\hline Asociación lineal por lineal & 4133.774 & 1 & .000 \\
\hline No. de casos válidos & 15305 & & \\
\hline
\end{tabular}

A. 1 casillas (8.3\%) tienen una frecuencia esperada inferior a 5. La frecuencia mínima esperada es de 3,71.

Fuente: Elaboración propia. SPSS Ver. 19. con base en la Primera Encuesta sobre Cultura Ciudadana 2010, Cuernavaca, Mor. 
El comportamiento que se encuentra distribuido un poco más uniforme, y reúne un mayor número de ocurrencias corresponde a encuestas que contestaron que percibían tanto el grado de control social y la inseguridad como medio y alto. Mediante esta tabla de contingencia se ilustra de mejor manera para inferir y comprobar que sí existe una relación entre la inseguridad y el control social del espacio en la ciudad de Cuernavaca (ver tabla 4).

La prueba de Chi cuadrado de Pearson arrojó un valor de 4851.127, que indica que tan solo un 8,3\% tuvo una frecuencia esperada menor a 5 de acuerdo con el modelo teórico, no obstante la mínima esperada con los cálculos del modelo en SPSS fue de 3,71, quiere decir, aún más baja que la sugerida por el modelo. Con 6 grados de libertad y con un nivel de significancia para ambos extremos de ,000. Si estos valores se comparan con los valores teóricos del modelo de 0,05, se concluye que existe una relación directa entre la inseguridad y el grado de control social del espacio y que tiene una significancia asintótica bilateral concluyente en la relación de los dos índices. La razón de verosimilitud, resultó ser significativa con un valor bilateral en las asíntotas de 0,000 ya que es menor a 0,05, con lo cual se puede aceptar que la presencia de un control social del espacio importante mejora sensiblemente la percepción de seguridad en los barrios. Pero surgen otras interrogantes sobre ¿cómo está relacionada?, ¿cuál es su tipo de relación?, para ello se aplicarán pruebas de correlación más adelante.
En lo que respecta a la relación que guarda la inseguridad con el grado de eficacia colectiva, se parte de la hipótesis nula que el grado de eficacia colectiva no tiene relación alguna con la inseguridad. En la tabla 5, se ilustran las frecuencias observadas y esperadas entre el grado de inseguridad y el grado de eficacia colectiva. En la estructura de la tabla 6 se observa además un peso enorme al interior de las dos categorías medio y muy alto, dejando los porcentajes bajos a las orillas o extremos. No obstante la prueba de significancia arroja un valor de 0,00 con lo cual se interpreta que las frecuencias proporcionadas por la muestra indican que el grado de inseguridad está significativamente relacionado con el grado de eficacia colectiva de la población de Cuernavaca. La hipótesis nula asume la no relación o dependencia entre la inseguridad y la eficacia colectiva. Para concluir que con lo observado en las tablas referidas tanto el control social del espacio y la eficacia colectiva están ligadas con la inseguridad en la ciudad de Cuernavaca, Morelos, de acuerdo con la población encuestada. Pero esta relación y significancia tendrán una mejor explicación en cuanto a la dependencia entre los tres índices. Por ello se hace necesario una prueba de correlación de Pearson, en la que se aplican las combinaciones de pruebas entre el grado de inseguridad, grado de control social del espacio y el grado de eficacia colectiva.

Si se observa la tabla 7 , en lo que respecta a la prueba de correlación grado de inseguridad, grado 
TABLA 5 GRADO DE INSEGURIDAD ^ GRADO DE EFICACIA COLECTIVA

\begin{tabular}{llrrrrr}
\multirow{2}{*}{ GRADO DE INSEGURIDAD } & \multicolumn{2}{c}{$\begin{array}{l}\text { GRADO DE EFICACIA COLECTIVA } \\
\text { MUY ALTO }\end{array}$} & \multicolumn{1}{c}{ ALTO } & MEDIO & BAJO & TOTAL \\
\multirow{2}{*}{ MUY ALTO } & Recuento & 0 & 0 & 62 & 0 & 62 \\
\cline { 2 - 7 } & Frecuencia esperada & 7 & 25.3 & 34.8 & 1.1 & 62.0 \\
\hline \multirow{2}{*}{ ALTO } & Recuento & 31 & 1991 & 5101 & 185 & 7308 \\
\cline { 2 - 7 } & Frecuencia esperada & 88.3 & 2984.1 & 4102.8 & 132.7 & 7308.0 \\
\hline \multirow{2}{*}{ MEDIO } & Recuento & 93 & 3707 & 3338 & 93 & 7231 \\
\cline { 2 - 7 } & Frecuencia esperada & 87.4 & 2952.7 & 4059.6 & 131.3 & 7231.0 \\
\hline \multirow{2}{*}{ BAJO } & Recuento & 61 & 552 & 92 & 0 & 705 \\
\cline { 2 - 7 } & Frecuencia esperada & 8.5 & 287.9 & 395.8 & 12.8 & 705.0 \\
\hline \multirow{2}{*}{ TOTAL } & Recuento & 185 & 6250 & 8593 & 278 & 15306 \\
\cline { 2 - 7 } & Frecuencia esperada & 185.0 & 6250.0 & 8593.0 & 278.0 & 15306.0 \\
\hline
\end{tabular}

Fuente: Elaboración propia. SPSS Ver. 19. con base en la Primera Encuesta sobre Cultura Ciudadana 2010, Cuernavaca, Morelos.

TABLA 6 PRUEBAS DE CHI CUADRADO EFICACIA COLECTIVA E INSEGURIDAD

\begin{tabular}{lrrr} 
PRUEBAS DE CHI CUADRADO & VALOR & GL & SIG. ASINTÓTICA BILATERAL \\
Chi-cuadrado de Pearson & $1823,619^{\mathrm{a}}$ & 9 & .000 \\
\hline Razón de verosimilitudes & 1757.667 & 9 & .000 \\
\hline Asociación lineal por lineal & 1578.542 & 1 & .000 \\
\hline No. de casos válidos & 15306 & & \\
\hline
\end{tabular}

A. 1 casillas (8.3\%) tienen una frecuencia esperada inferior a 5. La frecuencia mínima esperada es de 3,71.

Fuente: Elaboración propia. SPSS Ver. 19. con base en la Primera Encuesta sobre Cultura Ciudadana 2010, Cuernavaca, Morelos. 
TABLA 7 CORRELACIONES ENTRE LOS TRES INDICADORES

\begin{tabular}{llrrr} 
CORRELACIONES & & $\begin{array}{l}\text { GRADO DE } \\
\text { INSEGURIDAD }\end{array}$ & \multicolumn{1}{c}{$\begin{array}{l}\text { GRADO DE } \\
\text { EFICACIA } \\
\text { COLECTIVA }\end{array}$} & \multicolumn{2}{l}{$\begin{array}{l}\text { GRADO DE } \\
\text { CONTROL } \\
\text { SOCIAL }\end{array}$} \\
& & 1 &,$- 321^{* *}$ &,$- 520^{* *}$ \\
\hline & Correlación de Pearson & & .000 & .000 \\
\hline & Sig. (bilateral) & 15305 & 15305 & 15305 \\
\hline & $\mathrm{N}$ &,$- 321^{* *}$ & 1 &,$- 353^{* *}$ \\
\hline & Correlación de Pearson & .000 & & .000 \\
\hline GRADO DE EFICACIA COLECTIVA INSEGURIDAD & Sig. (bilateral) & 15305 & 15305 & 15305 \\
\hline & $\mathrm{N}$ &,$- 520^{* *}$ &,$- 353^{* *}$ & 1 \\
\hline & Correlación de Pearson & .000 & .000 & \\
\hline GRADO DE CONTROL SOCIAL & 15305 & 15305 & 15305
\end{tabular}

**. La correlación es significativa al nivel 0,01 (bilateral)

Fuente: Elaboración propia. SPSS Ver. 19. con base en la Primera Encuesta sobre Cultura Ciudadana 2010

de eficacia colectiva y grado de control social con ellos mismos, se encuentra una correlación cuadrada perfecta de 1 a 1 , en los 15,305, esto se debe a que las pruebas asumen una estructura en espejo, luego entonces correlacionar una variable con ella misma, sirve únicamente para corroborar que se incluya el total de la población encuestada y que el coeficiente $r$ de Pearson sea 1, comprueba que el método está bien aplicado y la base de datos se encuentra consistente.

El coeficiente de correlación $r$ de Pearson entre el grado de inseguridad y el grado de eficacia colectiva es igual a $(-, 321)$. El signo indica una correlación lineal negativa, lo cual quiere decir que en la ciudad de Cuernavaca la inseguridad es más alta cuando el grado de eficacia colectiva baja; mientras que el $r$ de Pearson entre inseguridad y control social del espacio es de (-,520), de igual manera existe una correlación lineal negativa, que se interpreta que la población encuestada percibe que en Cuernavaca la inseguridad aumenta cuando el grado de control social del espacio disminuye; con estas pruebas de independencia y correlación, se concluye que el control social del espacio tiene un mayor peso en la inseguridad que la eficacia colectiva: o de otro modo se puede interpretar que el control social 
del espacio tiene una relación más estrecha con la inseguridad.

Cabe mencionar que todas las pruebas de correlación entre los grados de inseguridad, control social del espacio y eficacia colectiva, arrojaron significancia absoluta al nivel de 0,000, que comprueba la validez de la encuesta levantada en cuanto al rigor de su tamaño, es decir, se rechaza la hipótesis nula de que el valor poblacional es cero. Con la nota de ser bilateral, con lo que se entiende que la probabilidad de que se obtengan resultados hacia un extremo $u$ otro entre inseguridad y control social del espacio, inseguridad y eficacia colectiva, está indefinido; es decir, que puede tener cualquiera de las dos direcciones, por eso es bilateral, es decir, no se especifica la dirección del efecto ${ }^{49}$. Se concluye con las pruebas de correlación aplicadas, que la inseguridad, el control social del espacio y la eficacia colectiva, están correlacionados significativamente mediante una relación negativa lineal directa, siendo el control social del espacio el que marca una pauta más estrecha con la inseguridad.

49 Como nota adicional, al comparar el modelo de correlación con los valores teóricos del coeficiente $r$ de Pearson, se pueden comparar con 0,05 cuando marca el SPSS con un asterisco y cuando lo marca con dos asteriscos, la comparación de sus niveles significativos lo hace con el valor teórico de 0,01 ; con lo que se podría pensar que el modelo es un poco mas exigente 0 detallado, pero esto varía de acuerdo a los criterios y necesidades estadísticas específicas.

\section{Conclusiones: el control social informal como factor determinante de la seguridad}

En el presente trabajo tomamos como punto de partida las reflexiones sobre las teorías ecológicas del delito, la desorganización social y la eficacia colectiva. Si bien esta última se basa en la idea de que existe una correlación entre la cohesión social entre los miembros de la comunidad aunada a su disposición para actuar en función del bien común y los niveles de violencia, nosotros cuestionamos la importancia que se atribuye a la relación existente entre la percepción de la inseguridad en comunidades específicas y la confianza mutua entre los residentes ${ }^{50}$. Si éste fuera el caso, en sitios donde se concentra la pobreza, el desempleo y la inseguridad inmobiliaria, la eficacia colectiva llegaría a servir como un mediador con respecto a la violencia.

El análisis multinivel de la muestra reveló que las colonias con altos niveles de eficacia colectiva se relacionan con una percepción de inseguridad

50 Sampson y Raudenbush, 1997. 
baja en las colonias, e incluso en demarcaciones en donde los índices delictivos son altos. De modo que dicha percepción es matizada por la confianza comunitaria que ésta genera, y sin embargo existen algunas colonias con un índice de eficacia colectiva alto, pero en donde la percepción de inseguridad puede llegar a ser alta cuando los índices delictivos son igualmente elevados. En otros casos en donde la eficacia colectiva y el control social eran bajos, la percepción de inseguridad fue alta aun cuando los índices delictivos fueran bajos. Por otra parte, en algunas de las colonias más peligrosas de la ciudad (Antonio Barona, Centro, Flores Magón) ${ }^{51}$, la percepción interna de inseguridad era baja, aun cuando existen eventos delictivos frecuentes. Entre los hallazgos de la investigación encontramos que cuando especificamente existen índices bajos de control social del espacio, esto afecta negativamente la percepción de la inseguridad del individuo.

En la presente investigación encontramos que un fuerte sentido comunitario se refleja en la eficacia colectiva y esto mejora la percepción de los residentes acerca de la seguridad aun cuando se trate de colonias tradicionalmente peligrosas. Sin embargo, encontramos algunas áreas con altos índices delictivos que no obstante, tienen un control social informal fuerte y una eficacia colectiva alta. En estos casos, los residentes no perciben una alta peligrosidad en su colonia o bien, puede ser indicativo de la existencia de un código de silencio, el cual inhibe el dar información relevante sobre actividades fuera de la ley. Por tanto, es posible sugerir que la percepción de inseguridad esté más relacionada con una baja eficacia colectiva y sentido comunitario que con los índices de crimen real.

El uso de análisis por correlaciones para desarrollar modelos para predecir la percepción de inseguridad utilizando una combinación de variables sociales, demográficas y físicas como detonadores potenciales arrojó resultados interesantes. Estos resultados sugieren que la reducción del crimen reduce solo parcialmente la percepción de inseguridad en los vecindarios urbanos. Para mejorar la percepción de inseguridad no es suficiente desarrollar un sentido de comunidad que fortalezca la eficacia colectiva entre los residentes así como la creación de un entorno construido que promueva la seguridad y reduzca el crimen. La encuesta demostró en cambio que la apropiación del territorio resulta determinante para atenuar la percepción de inseguridad en los vecindarios, lo cual enfatiza la importancia de acciones en el territorio, de que estamos frente a la espacialización de la problemática social actual.

Por una parte, las teorías ligadas a la sicología ambiental sugieren que las condiciones del entorno incitan el aumento de actos delictivos en el territorio

51 Información derivada de las Carpetas de Incidencia Delictiva del Municipio de Cuernavaca, 2006-2009. 
por lo que habría que diseñar espacios defensivos ${ }^{52}$ y evitar las ventanas rotas ${ }^{53}$ que se conviertan en signos de abandono y deterioro como un repliegue de control comunitario del espacio. Pero por la otra encontramos evidencias en la presente investigación de que existen colonias con entornos degradados así como altos índices delictivos y sin embargo, la percepción de la seguridad es baja e incluso, la cohesión social notablemente fuerte. Otro aspecto interesante, y que contradice las teorías de Jacobs ${ }^{54}$ acerca de la disminución de la delincuencia en presencia de distintos usos de suelo (dado que las áreas multifuncionales atraen un flujo continuo de gente que asegura una vigilancia informal) ya que en la colonia centro, la cual es eminentemente turística y sin embargo, reporta $400 \%$ más delitos que las colonias más peligrosas de la ciudad.

Uno de los hallazgos importantes del presente estudio es que la percepción de inseguridad se relaciona fuertemente con la falta de sentido comunitario o de eficacia colectiva que con los índices del crimen real. Además de que se registra una correlación todavía más significativa entre el control social informal y la percepción de la inseguridad que esta última con la eficacia colectiva. Estos resultados sugieren que para reducir el miedo al crimen y la percepción de vulnerabilidad de la población se deben realizar esfuerzos para desarrollar y fortalecer el sentido comunitario mediante la creación de entornos confiables y seguros.

Si bien al territorializar las actividades criminales, no son del todo evidentes los vínculos entre las características físicas de un vecindario y los índices de criminalidad registrados por la policía local, el control social informal es importante para la percepción de un entorno seguro porque se sustenta en un sentido territorial, de carácter instintivo. En cambio, la eficacia colectiva demanda una mayor vinculación social, la cual no se genera de manera natural en las comunidades y generalmente surge a partir de uno o más eventos que han involucrado las emociones de los residentes (miedo, ansiedad, odio, etc.). En el caso de barrios considerados como peligrosos existen complejos sistemas de vinculación, amistades y complicidades pero esto no implica necesariamente que se compartan los mismos valores por la generalidad. Para que una persona se sienta segura es necesario que desarrolle un sentido de apropiación del territorio de modo que se identifique con el entorno suficientemente como para defenderlo. En este sentido, la relación con el control social es más directa, personal y cotidiana.
52 Newman (1972).

53 Wilson y Kelling (1982).

54 Jacobs J., 1961. 
Por su parte, la eficacia colectiva podría estar relacionada con procesos sociales al margen de la ley y que sin embargo se encubren mediante redes sociales, vínculos interpersonales y un control social informal del espacio, el cual es efectivo para mantener la zona con un nivel de riesgo controlado. En contextos de alta incidencia delictiva pueden existir estrechos vínculos interpersonales, redes sociales consolidadas, o buena integración social, y no obstante, tener mecanismos de control social débiles o pocas posibilidades de incidir en los niveles de seguridad de la comunidad. Por tanto, la percepción que tiene la gente con respecto a su capacidad para controlar los eventos que afectan sus vidas incide sobre sus decisiones, sus aspiraciones, percepción sobre la propia vulnerabilidad, así como en su disposición a intervenir para lograr cambios significativos en su comunidad.

Aparentemente, la percepción de inseguridad tiene una estrecha correlación con el control social del espacio más que con la eficacia colectiva. Esto tiene una serie de implicaciones tales como el predominio de una territorialización del espacio, es decir, la apropiación y defensa del territorio juega un papel decisivo en la percepción de la inseguridad. Por tanto, para lograr una mayor percepción de seguridad -y con ello una mejor disposición a intervenir en la preservación del entorno- es necesario fomentar las prácticas de apropiación del espacio, es decir, las acciones enfocadas al mejoramiento de la calidad espacial de las comunidades (paseos peatonales, parques, mobiliario urbano, etc.). La eficacia colectiva acusa por tanto ciertos límites con relación a su capacidad para disminuir la percepción de inseguridad en las comunidades.

La violencia en América Latina está imponiendo lo que Arendt denomina una "sociedad totalizada", en donde el individuo es caracterizado por su soledad, falta de identidad y ausencia de relaciones sociales. Ante este panorama es importante referir la importancia de los controles formales e informales en la sociedad, en donde la gente adopta restricciones para poder estructurar sus relaciones con los demás y reducir así los costos de dichas interacciones. La estructura dominante se define mediante códigos de conducta, normas de comportamiento y convenciones sociales como restricciones informales acordadas de manera colectiva, tácita e implícita. Sin embargo, aun cuando las normas formales como las informales juegan un papel importante en el momento de tomar decisiones de carácter moral y ético, el resultado de la aplicación de dichas normas dependerá de cada sociedad en particular ${ }^{55}$.

55 North D.C., 1990:36 


\section{Bibliografía}

ARENDT, Hannah. On violence. New York and London, Harvest/HBJ Book. 1969. ISBN-10 0156695006.

BANDURA, Albert. Self-efficacy: the exercise of control. New York: Freeman.1997. 604 p. ISBN 9780-7167-2626-5.

BAUMAN, Zygmunt. Comunidad. En busca de seguridad en un mundo hostil. Madrid, España: Siglo XXI. 2003. 158 p. ISBN 978-84-323-1272-4.

BOHLE, Hans, DOWNING Thomas e.G., y WATTS Michel J. Climate Change and Social Vulnerability. Toward a Sociology and Geography of Food Insecurity. Global Environmental Change. 4(1): 37-48, 1994. ISSN 09593780.

BURSIK, Robert J. Social disorganization and theories of crime and delinquency: problems and prospects. Criminology. 26 (4): 519-552, 1988. ISSN 1745-9125.

CHAMBERS, Robert. Vulnerability, Coping and Policy. IDS Bulletin. 20(2):1-7, 1989. ISSN: 1759-5436.

COHEN, Stanley. Visiones del Control Social. Barcelona, Ediciones PPU. 1988. 416 p. ISBN 9788476652312.

DAVIS, Diane E. El factor Giuliani: delincuencia, la "cero tolerancia" en el trabajo policiaco y la transformación de la esfera pública en el centro de la Ciudad de México. Estudios Sociológicos. 25(3): 639-681, 2007. ISSN 0185-4186.
FELSON, Marcus. Routine activities and crime prevention in the developing metropolis. Criminology. 25(4): 911-31, 1987. ISSN 1745-9125.

FIREBAUGH, Glenn. The New Geography of Global Income Inequality. Cambridge MA, Harvard University Press. 2003. ISBN 0674010671.

FORER0, Juan. Colombia stepping up anti-drug training of Mexico's army, police. The Washington Post, Enero 21, 2011. ISSN 0190-8286.

GOODEY Jo. Boys dont cry. Masculinities, fear of crime and fearlessness. British Journal of Criminology. 37 (3): 401-418, 1997. ISSN 0007-0955.

GREENBERG, Stephanie, ROHE William M. y WILLIAMS Jay R. Safe and secure neighborhoods: Physical characteristics and informal territorial control in high and low crime neighborhoods. Washington, D.C.: U.S. Department of Justice, National Justice System. 1982. 179 p.

HURLEY Dan. On crime as a science. The New York Times. Enero 6, 2004. ISSN 0362-4331.

JACOBS, Jane. The Death and Life of Great American Cities. New York, The Modern Library. 1961. 600 p. ISBN 0-679-60047-7.

JANOWITZ, Morris. On Social Organization and Social Control. Chicago, Chicago University Press, 1991. 324 p. ISBN 0-226-39301-1.

JANOWITZ, Morris. Sociological Theory and Social Action. American Journal of Sociology. 81 (1): 82108, 1988. ISSN 0002-9602. 
KAUFMAN Susana G. Miedo, Perspectiva subjetivas y lazo social. En SCHMUCLER, Héctor. Miedos y memorias en las sociedades contemporáneas. Córdoba, Comunic-Arte Editorial. 2006. 323 p. ISBN 9789876020084

KORNHAUSER, Ruth R. Social Sources of Delinquency. Chicago, University of Chicago Press. 1978. 277 p. ISBN 0226451135

LAGUNAS Icela. Harán del DF la ciudad más vigilada del mundo. El gobierno local prevé concluir antes de 2010 una red de 8 mil cámaras en las 16 delegaciones. [En línea]. El Universal, Lunes 25 de febrero, 2008. [Fecha de consulta: 25 mayo 2011]. Disponible en: http://www.eluniversal.mx/ ciudad/89090.html.

LAWYERS Committee For Human Rights. Resumen de las recomendaciones del equipo de consultores encabezado por el ex-alcalde de Nueva York, Rudolph Giulani, Septiembre 4, 2003. [En línea]. Human Rights First. Disponible en: http://www. humanrightsfirst.org/wp-content/uploads/pdf/ ltr_Giuliani_recomm_090403_sp.pdf

LOGAN, John y MOLOTCH, Harvey. Urban Fortunes the political economy of place. Berkeley, University of California Press. 1987. ISBN 100520063414.

MORENOFF Jeffrey y SAMPSON Robert J. Violent crime and the spatial dynamic of neighborhood transition: Chicago 1970-1990. Social Forces. 76 31-64, 1997. ISSN 0037-7732.
NEWMAN, Oscar. Defensible space. Crime prevention through urban design. London, Macmillan. 1972. ISBN 100020007507.

NORTH Douglass C. Institutions, Institutional Change and Economic Performance. New York, Cambridge University Press. 1990. ISBN 100521397340.

PATILLO-MC COY, Mary E. Black Picket Fences: priviledge and peril among the Black middle class. Chicago, Chicago University Press. 1999. ISBN100226649296

PATILLO-MC COY, Mary E. Sweet mothers and Gangbangers: Managine Crime in a Black Middle-Class Neighborhood. Social Forces. (76) 747-74, 1988. ISSN 0037-7732.

SAMPSON Robert J. y GROVES W. Byron. Community Structure and crime: testing social disorganization theory. American Journal of Sociology. 94: $774-$ 802, 1989. ISSN: 0002-9602.

SAMPSON Robert J., MORENOFF Jeffrey D., y EARLS Felton. "Beyond social capital: spatial dynamics of collective efficacy for children. [En línea] American Sociological Review. 64(5): 633-660, 1999. ISSN 0003-1224. Disponible en: http://scholar. harvard.edu/sampson/publications/beyond-social-capital-spatial-dynamics-collective-efficacychildren-0.

SAMPSON Robert J., MORENOFF Jeffrey D., y EARLS Felton. Neighborhoods and violent crime: A multilevel study off collective efficacy. Science. (277): 918-924, 1996. ISSN 0036-8075. 
SCHLOSSER, Eric. The Prison-Industrial Complex. [En línea] The Atlantic Monthly. December, 1998. ISSN: 1072-7825. Disponible en: http://www theatlantic.com/magazine/archive/1998/12/theprison-industrial-complex/4669/

UTE Gabriel y GREVE Werner. The psychology of fear of crime. Conceptual and methodological perspectives. The British Journal of Criminology. 43(3):600614, 2003. ISSN 0007-0955.

VALENZUELA Aguilera, Alfonso (coord). Ciudades Seguras. Cultura Ciudadana, Eficacia Colectiva y Control Social del Espacio. México, Miguel Angel Porrúa Editores. 2011. 328 p. ISBN 978-607-401447-1.

VALENZUELA Aguilera, Alfonso. City of fear: The social control of urban space in Latin America. Tria$\log$ (87): 25-30, 2005. ISSN 0724-6234.
WACQUANT, Loïc. La penalización de la miseria. De la importación de políticas de seguridad. Renglones. 51: 6-11, 2002. ISSN 0186-4963.

WILSON, James 0. y KELLING George L. Broken Windows: Police and Neighborhood Safety. The Atlantic Monthly. 249: 29-38, 1982. ISSN 1072-7825.

WILSON, William Julius. When Work Disappears. The World of the new urban poor. New York, Knopf. 1996. 322 p. ISBN 0-394-57935-6.

WRIGHT MILLS, Charles. The Power Elite. New York, Oxford University Press. 1956. ISBN 019 500680-1.

WURFF, Adri Van Der, STRINGER, Peter y TIMMER F. Feelings of unsafety in residential surroundings. En: CANTER, David, JESUINO, Jorge y SOCZCA, Lums (eds) Environmental Social Psychology. The Hague Kluwer. 1988. p. 135-148. ISBN 9024737060. 